BMJ Open

Diabetes

Research

\& Care

\section{Real-world evidence on sodium-glucose cotransporter-2 inhibitor use and risk of Fournier's gangrene}

To cite: Yang JY, Wang T, Pate V, et al. Real-world evidence on sodium-glucose cotransporter-2 inhibitor use and risk of Fournier's gangrene. BMJ Open Diab Res Care 2020;8:e000985. doi:10.1136/ bmjdrc-2019-000985

Received 19 0ctober 2019 Revised 27 November 2019 Accepted 10 December 2019

\section{Check for updates}

(c) Author(s) (or their employer(s)) 2020. Re-use permitted under CC BY-NC. No commercial re-use. See rights and permissions. Published by BMJ.

${ }^{1}$ Department of Epidemiology, University of North Carolina at Chapel Hill, Chapel Hill, North Carolina, USA

2Department of Medicine, University of North Carolina at Chapel Hill, Chapel Hill, North Carolina, USA

Correspondence to Jeff Yufeng Yang; jeff.yang@unc.edu

\section{ABSTRACT}

Background Sodium-glucose cotransporter-2 inhibitors (SGLT2i) have been associated with increased occurrence of Fournier's gangrene ( $\mathrm{FG})$, a rare but serious form of necrotizing fasciitis, leading to a warning from the Food and Drug Administration. Real-world evidence on FG is needed to validate this warning.

Methods We used data from IBM MarketScan (2013-2017) to compare the incidence of $F G$ among adult patients who initiated either SGLT2i, a dipeptidyl peptidase-4 inhibitor (DPP4i), or any non-SGLT2i antihyperglycemic medication. FG was defined using inpatient International Classification of Diseases, Ninth Edition and Tenth Edition diagnosis codes 608.83 and N49.3, respectively, combined with procedure codes for debridement, surgery, or systemic antibiotics. We estimated crude incidence rates (IRs) using Poisson regression, and crude and adjusted HRs (aHR) and 95\% Cls using standardized mortality ratio-weighted Cox proportional hazards models. Sensitivity analyses examined the impact of alternative outcome definitions. Results We identified 211671 initiators of SGLT2i $(n=93197)$ and DPP4i $(n=118474)$, and 305329 initiators of SGLT2i ( $n=32868)$ and non-SGLT2i $(n=272461)$. Crude FG IR ranged from 3.2 to 3.8 cases per 100000 personyears during a median follow-up of $0.51-0.58$ years. Compared with DPP4i, SGLT2i initiation was not associated with increased risk of $\mathrm{FG}$ for any outcome definition, with aHR estimates ranging from $0.25(0.04-1.74)$ to 1.14 (0.86-1.51). In the non-SGLT2i comparison, we observed an increased risk of FG for SGLT2i initiators when using FG diagnosis codes alone, using all diagnosis settings (aHR $1.80 ; 0.53-6.11$ ) and inpatient diagnoses only (aHR 4.58; $0.99-21.21$ ).

Conclusions No evidence of increased risk of FG associated with SGLT2i was observed compared with DPP4i, arguably the most relevant clinical comparison. However, uncertainty remains based on potentially higher risk in the broader comparison with all non-SGLT2i antihyperglycemic agents and the rarity of $F G$. Trial registration number EUPAS Register Number 30018.

\section{INTRODUCTION}

In August 2018, the US Food and Drug Administration (FDA) released a safety warning linking sodium-glucose cotransporter-2 inhibitors (SGLT2i), the newest

\section{Significance of this study}

What is already known about this subject?

- Sodium-glucose cotransporter-2 inhibitors (SGLT2i), the newest class of antihyperglycemic medications, have been linked to an increased occurrence of Fournier's gangrene, a rare, necrotizing fasciitis of the perineum, using data from the Food and Drug Administration (FDA) Adverse Event Reporting System, and an FDA warning was issued in response to this finding.

What are the new findings?

- Using administrative data from the commercially insured US population and an active-comparator, new-user cohort study design, we found no difference in risk of Fournier's gangrene between patients initiating SGLT2i and patients initiating dipeptidyl peptidase-4 inhibitors, a similar branded secondline antihyperglycemic medication class.

How might these results change the focus of research or clinical practice?

- This study suggests that patients who are prescribed SGLT2i in real-world practice may not be at increased risk for Fournier's gangrene compared with patients who are prescribed similar secondline, branded antihyperglycemic medications.

- Given the very low incidence of Fournier's gangrene in the USA, this evidence must be weighed against the clinical benefits of SGLT2i in patients with type 2 diabetes mellitus.

class of antihyperglycemic medications, to an increased occurrence of Fournier's gangrene (FG), a rare, necrotizing fasciitis of the perineum. ${ }^{1}$ Despite overall low incidence in the USA ( 1.6 cases per 100000 male patients), ${ }^{2}$ FG is often accompanied by poor management options and prognosis ${ }^{3}$ and results in debilitating complications and disfigurement in most infected patients. Approximately $7.5 \%$ of patients with FG die. ${ }^{24-6}$

The FDA warning, which attracted media attention, ${ }^{7}$ was based on $12 \mathrm{FG}$ cases $(7 \mathrm{men}$, 5 women) reported through the FDA Adverse 
Event Reporting System (FAERS) from 2013 to 2018, as well as individual case reports in the medical literature. ${ }^{8-10}$ A more recent review of the FAERS and case reports yielded similar conclusions based on 55 FG cases among patients receiving SGLT2i from 2013 to 2019, compared with 19 FG cases among patients receiving other antihyperglycemic medications from 1984 to 2019. ${ }^{11}$ However, the structure and quality of FAERS reporting precluded any ability to estimate comparative incidence or establish causality. ${ }^{12}$

To address these limitations and to validate evidence behind the FDA warning in a real-world setting, we evaluated the association between SGLT2i initiation and FG risk in a large healthcare administrative claims database from the commercially insured US population, using an active-comparator, new-user (ACNU) study design. ${ }^{13}$

\section{METHODS}

\section{Data source}

We used data from IBM MarketScan from 1 January 2012 to 31 December 2017. The base population consisted of all patients with $\geq 1$ prescription dispensing claim for an SGLT2i or dipeptidyl peptidase-4 inhibitor (DPP4i) between 1 April 2013 (US market entry date of canagliflozin ${ }^{14}$ ) and 30 June 2017 (to allow at least 6 months of follow-up), identified using national drug codes (NDCs). Eligible patients were adults aged 18-64 years with $\geq 12$ months of continuous enrollment in MarketScan prior to first eligible prescription dispensing claim without a prescription for either SGLT2i or DPP4i (washout period to define new use). The study protocol was registered with the European Network of Centres for Pharmacoepidemiology and Pharmacovigilance on 5 June 2019 (http://www.encepp.eu/encepp/viewResource.htm?id= 30019).

\section{Exposure definitions}

Exposure to a study drug was defined by $\geq 2$ same-drug class prescription dispensing claims of either an SGLT2i or DPP4i within a predefined 'prescription window' (first prescription's recorded days' supply plus a 30-day grace period). The second prescription served as the index date for the analysis. This two-prescription approach restricts the analysis to a cohort for whom we are more confident were taking the cohort drugs, compared with cohorts requiring just a single prescription. We excluded patients who received a comparator drug (including the empagliflozin-linagliptin combination drug) prior to or on the index date.

\section{Outcome definitions}

Fournier's gangrene was defined using the International Classification of Diseases, Ninth Edition and Tenth Edition (ICD-9 and ICD-10) diagnosis codes 608.83 ('vascular disorders, including Fournier's disease') and N49.3 ('Fournier's gangrene'), respectively. Patients who received an FG diagnosis code in the 12 months prior to the index date were excluded from analysis. To increase outcome specificity, we required FG diagnoses to either occur in an inpatient setting or be followed by a hospitalization within 7 days, and be additionally accompanied by systemic antibiotic, debridement, or related surgical procedure $^{4}$ within 7 days (primary outcome definition). Codes were informed through prior literature ${ }^{15-25}$ and clinical guidance.

Acknowledging the lack of standard, validated claimsbased definitions for FG, and to examine the possible impact of outcome misclassification in a rare outcome setting, we considered less stringent (higher sensitivity, reduced specificity) secondary outcome definitions. First, we removed the systemic antibiotic requirement (as NDCs may not be billed during inpatient stays) and considered only debridement and surgical procedures. Second, we applied various diagnosis-only definitions, which have typically been used in published literature. ${ }^{245}$

We further identified and evaluated additional ICD-9 and ICD-10 diagnosis codes with a forward-backward mapping approach using the General Equivalence Mappings crosswalk provided by the Centers for Medicare and Medicaid Services. ${ }^{26}$ Finally, because there are no specific codes for FG in women, we used a combination of codes $(785.4,616.3,616.4$, N75.1, N76.4, I96) used in previous epidemiologic study of FG. ${ }^{245}$

\section{Statistical analysis}

Follow-up for outcomes started at the index date (date of the second prescription) and was censored at the first occurrence of either FG, treatment discontinuation, a prescription of the comparator drug class (all cohorts), disenrollment, or 31 December 2017.

We estimated propensity scores using multivariable logistic regression to control for measured confounding, with baseline covariates (patient demographics, comorbidities, risk factors for $\mathrm{FG},{ }^{561012}$ medication use history, and baseline healthcare utilization) measured in the 12 months prior to index date. All propensity score analyses were performed separately for patients with index dates before versus after 1 October 2015, to account for possible billing changes in the ICD-9 versus ICD-10 eras. Covariate balance was assessed using standardized mean differences. ${ }^{27}$

We estimated the average treatment effect in the treated by standardizing the distribution of measured covariates to the SGLT2i cohorts using standardized mortality ratio (SMR) weighting. ${ }^{28}$ We estimated crude incidence rates (IRs) using Poisson regression, and crude and adjusted HRs (aHR) and 95\% CIs using SMRweighted Cox proportional hazards models. All analyses were performed using SAS V.9.4.

\section{Sensitivity analyses}

To reflect the comparison made in the FAERS analysis, we broadened the comparator group to include all nonSGLT2i antihyperglycemic medications (sulfonylureas, thiazolidinediones, DPP4i, glucagon-like peptide-1 receptor agonists, and insulins). In this analysis, we 
Table 1 Key baseline characteristics of eligible initiators of SGLT2i, compared with initiators of DPP4i and non-SGLT2i antihyperglycemic medications, before and after implementation of SMR weighting* (365-day washout period)

\begin{tabular}{|c|c|c|c|c|c|c|}
\hline \multirow{2}{*}{$\begin{array}{l}\text { Characteristics } \\
\text { (measured 1 year ( } 365 \\
\text { days) prior to date of } \\
\text { cohort drug initiation) }\end{array}$} & \multicolumn{3}{|c|}{ SGLT2i vs DPP4i } & \multicolumn{3}{|c|}{ SGLT2i vs non-SGLT2i } \\
\hline & $\begin{array}{l}\text { SGLT2i } \\
\text { initiators } \\
(n=93197+)\end{array}$ & $\begin{array}{l}\text { DPP4i initiators } \\
(n=118474)\end{array}$ & $\begin{array}{l}\text { Weighted DPP4i } \\
\text { initiators } \\
(n=94322)\end{array}$ & $\begin{array}{l}\text { SGLT2i } \\
\text { initiators } \\
(n=32868+)\end{array}$ & $\begin{array}{l}\text { Non-SGLT2i } \\
\text { initiators } \\
(n=272461)\end{array}$ & $\begin{array}{l}\text { Weighted non- } \\
\text { SGLT2i initiators } \\
(n=32942)\end{array}$ \\
\hline Age, mean (SD) & $52.2(8.3)$ & $52.9(8.2)$ & $52.2(8.3)$ & $51.3(8.6)$ & $49.8(10.3)$ & $51.3(8.6)$ \\
\hline Male & $50018(53.7)$ & $66025(55.7)$ & $48811(51.7)$ & $16870(51.3)$ & $135938(49.9)$ & $16945(51.4)$ \\
\hline \multicolumn{7}{|l|}{ Diabetes comorbidities } \\
\hline Nephropathy & $4422(4.7)$ & $5298(4.5)$ & $4485(4.8)$ & $748(2.3)$ & $8052(3.0)$ & $748(2.3)$ \\
\hline Neuropathy & 9197 (9.9) & $8156(6.9)$ & $9460(10.0)$ & $1604(4.9)$ & $13028(4.8)$ & $1609(4.9)$ \\
\hline Retinopathy & $6895(7.4)$ & $6265(5.3)$ & $6724(7.1)$ & $1186(3.6)$ & $9274(3.4)$ & $1185(3.6)$ \\
\hline $\begin{array}{l}\text { Peripheral vascular } \\
\text { disease }\end{array}$ & $1387(1.5)$ & $1978(1.7)$ & $1412(1.5)$ & $359(1.1)$ & $3452(1.3)$ & $361(1.1)$ \\
\hline \multicolumn{7}{|l|}{ General health comorbidities } \\
\hline Obesity & $25256(27.1)$ & $24921(21.0)$ & $26087(27.7)$ & $8411(25.6)$ & $60653(22.3)$ & $8461(25.7)$ \\
\hline Alcohol abuse & $693(0.7)$ & $1075(0.9)$ & $701(0.7)$ & $254(0.8)$ & $3526(1.3)$ & $255(0.8)$ \\
\hline COPD & $10374(11.1)$ & $13484(11.4)$ & $10791(11.4)$ & 3795 (11.5) & $31138(11.4)$ & $3812(11.6)$ \\
\hline Liver disease & $633(0.7)$ & $897(0.8)$ & $655(0.7)$ & $165(0.5)$ & $2328(0.9)$ & $165(0.5)$ \\
\hline Renal disease & $3218(3.5)$ & $6824(5.8)$ & $3381(3.6)$ & $650(2.0)$ & $12481(4.6)$ & $646(2.0)$ \\
\hline Chronic kidney disease & $2642(2.8)$ & $5991(5.1)$ & $2787(3.0)$ & $553(1.7)$ & $10874(4.0)$ & $550(1.7)$ \\
\hline History of cancer & $4217(4.5)$ & $6316(5.3)$ & $4302(4.6)$ & $1422(4.3)$ & $13479(4.9)$ & $1421(4.3)$ \\
\hline Hypertension & $66737(71.6)$ & $81683(68.9)$ & $67919(72.0)$ & $21880(66.6)$ & $164202(60.3)$ & $21966(66.7)$ \\
\hline Congestive heart failure & $2383(2.6)$ & 3889 (3.3) & $2381(2.5)$ & $604(1.8)$ & $8546(3.1)$ & $603(1.8)$ \\
\hline $\begin{array}{l}\text { Acute myocardial } \\
\text { infarction }\end{array}$ & $1572(1.7)$ & $2343(2.0)$ & $1551(1.6)$ & $410(1.2)$ & $5296(1.9)$ & $412(1.2)$ \\
\hline Cerebrovascular disease & 3226 (3.5) & $4747(4.0)$ & $3181(3.4)$ & $899(2.7)$ & $9959(3.7)$ & $894(2.7)$ \\
\hline Depression & $9996(10.7)$ & $11560(9.8)$ & $10569(11.2)$ & $3432(10.4)$ & $28693(10.5)$ & $3453(10.5)$ \\
\hline Smoking & $4739(5.1)$ & $6739(5.7)$ & $4845(5.1)$ & $1619(4.9)$ & $17018(6.2)$ & $1623(4.9)$ \\
\hline Dyslipidemia & $67685(72.6)$ & $79944(67.5)$ & $68433(72.6)$ & $21698(66.0)$ & $148508(54.5)$ & $21817(66.2)$ \\
\hline Ischemic heart disease & $9358(10.0)$ & $11794(10.0)$ & 9364 (9.9) & $2533(7.7)$ & $22318(8.2)$ & $2543(7.7)$ \\
\hline \multicolumn{7}{|l|}{ Prior medication use } \\
\hline Statins & $58728(63.0)$ & $71108(60.0)$ & $58907(62.5)$ & $17497(53.2)$ & $124490(45.7)$ & $17593(53.4)$ \\
\hline ACEI & $41685(44.7)$ & $52489(44.3)$ & $42320(44.9)$ & $12321(37.5)$ & $98889(36.3)$ & 12367 (37.5) \\
\hline $\begin{array}{l}\text { Angiotensin receptor } \\
\text { blockers }\end{array}$ & $25726(27.6)$ & $30146(25.4)$ & $26040(27.6)$ & $8275(25.2)$ & $52913(19.4)$ & $8332(25.3)$ \\
\hline Beta blockers & $27084(29.1)$ & $34111(28.8)$ & $27583(29.2)$ & $8697(26.5)$ & $78305(28.7)$ & 8679 (26.3) \\
\hline $\begin{array}{l}\text { Calcium channel } \\
\text { blockers }\end{array}$ & $18391(19.7)$ & $24437(20.6)$ & $18564(19.7)$ & 5869 (17.9) & 48854 (17.9) & 5876 (17.8) \\
\hline Loop diuretics & $6203(6.7)$ & $7763(6.6)$ & $6584(7.0)$ & $1645(5.0)$ & $15660(5.7)$ & $1643(5.0)$ \\
\hline Non-loop diuretics & $31322(33.6)$ & 38517 (32.5) & $32105(34.0)$ & 10608 (32.3) & $77330(28.4)$ & $10650(32.3)$ \\
\hline Aspirin & $5146(5.5)$ & $5691(4.8)$ & $5412(5.7)$ & $1092(3.3)$ & $11438(4.2)$ & $1089(3.3)$ \\
\hline Glucocorticoids & $16867(18.1)$ & 21037 (17.8) & $17186(18.2)$ & $6664(20.3)$ & $51214(18.8)$ & $6690(20.3)$ \\
\hline Metformin & 74749 (80.2) & $101535(85.7)$ & 74988 (79.5) & $25985(79.1)$ & 185703 (68.2) & $26133(79.3)$ \\
\hline Sulfonylureas & 30026 (32.2) & 40174 (33.9) & 30929 (32.8) & - & - & - \\
\hline GLP-1 receptor agonist & $23321(25.0)$ & $6122(5.2)$ & $24966(26.5)$ & - & - & - \\
\hline Thiazolidinediones & 7717 (8.3) & $8059(6.8)$ & $8278(8.8)$ & - & - & - \\
\hline Insulin (long-acting) & 26276 (28.2) & 14893 (12.6) & $27593(29.3)$ & - & - & - \\
\hline
\end{tabular}

Measures of healthcare utilization in the year prior to index date

Number of $\mathrm{HbA} 1 \mathrm{c}$ tests in the past year

$7355(7.9)$

$12730(10.7)$

$7429(7.9)$

$3176(9.7)$

$57820(21.2)$

$3717(11.3)$ 
Table 1 Continued

\begin{tabular}{|c|c|c|c|c|c|c|}
\hline \multirow{2}{*}{$\begin{array}{l}\text { Characteristics } \\
\text { (measured } 1 \text { year (365 } \\
\text { days) prior to date of } \\
\text { cohort drug initiation) }\end{array}$} & \multicolumn{3}{|c|}{ SGLT2i vs DPP4i } & \multicolumn{3}{|c|}{ SGLT2i vs non-SGLT2i } \\
\hline & $\begin{array}{l}\text { SGLT2i } \\
\text { initiators } \\
(n=93197 t)\end{array}$ & $\begin{array}{l}\text { DPP4i initiators } \\
(n=118474)\end{array}$ & $\begin{array}{l}\text { Weighted DPP4i } \\
\text { initiators } \\
(n=94322)\end{array}$ & $\begin{array}{l}\text { SGLT2i } \\
\text { initiators } \\
(n=32868+)\end{array}$ & $\begin{array}{l}\text { Non-SGLT2i } \\
\text { initiators } \\
(\mathrm{n}=\mathbf{2 7 2 4 6 1 )}\end{array}$ & $\begin{array}{l}\text { Weighted non- } \\
\text { SGLT2i initiators } \\
(n=32942)\end{array}$ \\
\hline 1 & $19992(21.5)$ & $31750(26.8)$ & $20222(21.4)$ & $9902(30.1)$ & 89693 (32.9) & 9199 (27.9) \\
\hline 2 & $27039(29.0)$ & 34897 (29.5) & $27504(29.2)$ & $9993(30.4)$ & $67450(24.8)$ & $9636(29.3)$ \\
\hline 3 & $38811(41.6)$ & 39097 (33.0) & $39168(41.5)$ & $9797(29.8)$ & $57498(21.1)$ & $10390(31.5)$ \\
\hline $\begin{array}{l}\text { Influenza shot received in } \\
\text { the past year }\end{array}$ & $29985(32.2)$ & $36648(30.9)$ & 30500 (32.3) & $9754(29.7)$ & 77115 (28.3) & $9779(29.7)$ \\
\hline \multicolumn{7}{|l|}{ Number of hospitalizations } \\
\hline 0 & 86515 (92.8) & $107154(90.4)$ & $87356(92.6)$ & $31113(94.7)$ & 237877 (87.3) & $31201(94.7)$ \\
\hline 1 & $5540(5.9)$ & $8822(7.4)$ & $5791(6.1)$ & $1507(4.6)$ & $27291(10.0)$ & $1497(4.5)$ \\
\hline 2 & $850(0.9)$ & $1694(1.4)$ & $876(0.9)$ & $189(0.6)$ & $4858(1.8)$ & $183(0.6)$ \\
\hline$\geq 3$ & $292(0.3)$ & $804(0.7)$ & $299(0.3)$ & $59(0.2)$ & $2435(0.9)$ & $62(0.2)$ \\
\hline \multicolumn{7}{|c|}{ Number of emergency department visits } \\
\hline 0 & $74332(79.8)$ & $92328(77.9)$ & $74773(79.3)$ & $26767(81.4)$ & $203129(74.6)$ & $26847(81.5)$ \\
\hline 1 & $12886(13.8)$ & $17430(14.7)$ & $13138(13.9)$ & $4333(13.2)$ & $45534(16.7)$ & $4325(13.1)$ \\
\hline$\geq 2$ & $5979(6.4)$ & $8716(7.4)$ & $6411(6.8)$ & $1768(5.4)$ & $23798(8.7)$ & $1770(5.4)$ \\
\hline \multicolumn{7}{|c|}{ Number of physician encounters } \\
\hline 0 & $322(0.3)$ & $671(0.6)$ & $329(0.3)$ & $154(0.5)$ & $2703(1.0)$ & $153(0.5)$ \\
\hline $1-3$ & $6798(7.3)$ & $12176(10.3)$ & $6747(7.2)$ & 3269 (9.9) & $38247(14.0)$ & $3263(9.9)$ \\
\hline $4-6$ & $15455(16.6)$ & $22137(18.7)$ & $15477(16.4)$ & $6250(19.0)$ & $51111(18.8)$ & $6267(19.0)$ \\
\hline$\geq 7$ & $70622(75.8)$ & $83490(70.5)$ & $71770(76.1)$ & 23195 (70.6) & $180400(66.2)$ & 23259 (70.6) \\
\hline
\end{tabular}

*Weighted by standardizing the comparator drug initiators to the population of SGLT2i initiators, using the propensity score odds (PS/(1-PS)), to estimate the average treatment effect in the treated (ATT).

†The number of eligible SGLT2i initiators is different between comparisons due to the washout period exclusion used to define new use of the study drugs in each pairwise comparison. For the SGLT2i vs DPP4i comparison, we exclude patients who were prevalent users of either drug at baseline. For the SGLT2i vs non- SGLT2i comparison, we exclude patients who were prevalent users of any antidiabetic drug at baseline, which results in a much greater number of patients being excluded at baseline. For example, an SGLT2i initiator who receives sulfonylurea during the baseline period is included in the SGLT2i vs DPP4i comparison, but not in the SGLT2i vs non- SGLT2i comparison. Therefore, the SGLT2i vs DPP4i comparison cohort is not a strict subset of the SGLT2i vs non-SGLT2i comparison cohort. This approach is common and seeks to emulate the drug initiation protocol in a randomized trial.

ACEI, ACE inhibitor; COPD, chronic obstructive pulmonary disease; DPP4i, dipeptidyl peptidase-4 inhibitors; GLP, glucagon-like peptide; HbA1c, hemoglobin A1c; SGLT2i, sodium-glucose cotransporter-2 inhibitors; SMR, standardized mortality ratio.

excluded all patients with baseline use of any of these antihyperglycemic medications to preserve new-user cohorts.

\section{RESULTS}

Eligible cohort

We identified 211671 eligible new users of SGLT2i $(\mathrm{n}=93197)$ and DPP4i $(\mathrm{n}=118474)$ and 305329 eligible new users of SGLT2i $(n=32868)$ and non-SGLT2i antihyperglycemic medications $(\mathrm{n}=272461)$ (table 1 ; see footnote regarding difference in SGLT2i new-user counts between comparisons). Crude FG IR (primary definition) for the SGLT2i versus DPP4i comparison was 3.8 (95\% CI 1.2 to 11.8 ) vs 3.2 (95\% CI 1.0 to 9.9) per 100000 personyears during a median follow-up of 0.58 (IQR, 0.27-1.16) and 0.51 (IQR, $0.25-1.05$ ) years, respectively.

\section{SGLT2i vs DPP4i}

For all outcome definitions, the aHR of FG did not increase for SGLT2i initiators compared with DPP4i initiators (figure 1). Estimates ranged from 0.25 (95\% CI 0.04 to 1.74) to 0.76 (95\% CI 0.12 to 4.97 ) when using ICD codes 608.83 and N49.3 only, and 0.58 (95\% CI 0.11 to 3.04 ) to 1.14 (95\% CI 0.86 to 1.51 ) when using expanded definitions. However, CIs were wide due to the low number of events and there was noticeable change-in-estimate between crude and adjusted HR estimates (figure 1).

\section{SGLT2i vs non-SGLT2i}

We observed an increased risk of FG for SGLT2i initiators compared with initiators of non-SGLT2i antihyperglycemic medications when using FG diagnosis codes only, using all diagnostic settings (aHR 1.80; 95\% CI 0.53 to 6.11 ) and inpatient diagnoses only (aHR 4.58; $95 \%$ CI 0.99 to 21.21) (figure 1). When requiring debridement, surgery, or antibiotics within 7 days of diagnosis, we observed zero FG events in the SGLT2i cohort arm. Expanded diagnosis definitions yielded aHR estimates closer to the null, ranging from 0.66 (95\% CI 0.20 to 2.16 ) to 1.71 (95\% CI 0.66 to 4.46 ). 


\begin{tabular}{|c|c|c|c|c|c|c|}
\hline \multirow[b]{2}{*}{ Outcome } & \multicolumn{2}{|c|}{ Events } & \multirow{2}{*}{$\begin{array}{l}\text { Crude } \\
\text { HR }(95 \% \mathrm{Cl})\end{array}$} & \multirow{2}{*}{$\begin{array}{l}\text { Adjusted } \\
\text { HR }(95 \% \mathrm{Cl})\end{array}$} & \multirow[b]{2}{*}{ Favors SGLT2i } & \multirow[b]{2}{*}{ Favors DPP4i } \\
\hline & SGLT2i & DPP4i & & & & \\
\hline Def1 & 7 & 9 & $0.95(0.35,2.54)$ & $0.49(0.11,2.15)$ & & \\
\hline Def2 & 6 & 4 & $1.82(0.51,6.47)$ & $0.51(0.09,2.94)$ & & \\
\hline Def3 & 134 & 131 & $1.22(0.96,1.55)$ & $1.01(0.73,1.39)$ & & \\
\hline Def4 & 39 & 34 & $1.36(0.86,2.15)$ & $0.90(0.45,1.82)$ & & \\
\hline Def5 & 180 & 166 & $1.29(1.05,1.60)$ & $1.14(0.86,1.51)$ & & \\
\hline Def6 & 52 & 42 & $1.48(0.99,2.23)$ & $1.05(0.56,1.94)$ & & \\
\hline Def7 & 3 & 3 & $1.21(0.25,5.88)$ & $0.25(0.04,1.74)$ & & \\
\hline Def9 & 23 & 23 & $1.18(0.67,2.10)$ & $0.63(0.27,1.45)$ & & \\
\hline Def11 & 31 & 29 & $1.27(0.77,2.11)$ & $0.77(0.36,1.65)$ & & \\
\hline Def13 & 0 & 1 & & & & \\
\hline Def14 & 2 & 5 & $0.47(0.10,2.30)$ & $0.58(0.11,3.04)$ & & \\
\hline Def15 & 5 & 9 & $0.66(0.22,1.97)$ & $0.85(0.26,2.76)$ & & \\
\hline Def16 & 2 & 2 & $1.20(0.18,8.08)$ & $0.76(0.12,4.97)$ & & \\
\hline Def17 & 13 & 17 & $0.90(0.44,1.83)$ & $0.75(0.30,1.83)$ & & \\
\hline \multirow[t]{3}{*}{ Def18 } & 19 & 22 & $1.02(0.55,1.88)$ & $0.93(0.43,2.04)$ & & \\
\hline & & & & & $T$ & $T$ \\
\hline & & & & & .2 & 5 \\
\hline \multirow[b]{3}{*}{ Outcome } & \multirow{2}{*}{\multicolumn{2}{|c|}{ Events }} & & & \multirow{2}{*}{\multicolumn{2}{|c|}{$\operatorname{aHR}(95 \% \mathrm{Cl})$}} \\
\hline & & & Crude & Adjusted & & \\
\hline & SGLT2i & Non-SGLT2i & $\mathrm{HR}(95 \% \mathrm{Cl})$ & $\mathrm{HR}(95 \% \mathrm{Cl})$ & Favors SGLT2i & Favors Non-SGLT2i \\
\hline Def1 & 3 & 21 & $1.27(0.38,4.22)$ & $1.80(0.53,6.11)$ & & \\
\hline Def2 & 2 & 8 & $2.49(0.55,11.40)$ & $4.58(0.99,21.21)$ & & \\
\hline Def3 & 33 & 351 & $0.81(0.57,1.16)$ & $0.90(0.63,1.30)$ & & \\
\hline Def4 & 9 & 106 & $0.75(0.38,1.49)$ & $1.04(0.52,2.08)$ & & \\
\hline Def5 & 44 & 426 & $0.89(0.65,1.21)$ & $0.99(0.72,1.36)$ & & \\
\hline Def6 & 11 & 120 & $0.81(0.44,1.50)$ & $1.10(0.59,2.08)$ & & \\
\hline Def7 & 0 & 5 & & & & \\
\hline Def9 & 3 & 54 & $0.48(0.15,1.54)$ & $0.66(0.20,2.16)$ & & \\
\hline Def11 & 5 & 62 & $0.70(0.28,1.73)$ & $0.94(0.37,2.38)$ & & \\
\hline Def13 & 0 & 0 & & & & \\
\hline Def14 & 1 & 10 & $0.83(0.10,6.55)$ & $1.02(0.12,8.39)$ & & \\
\hline Def15 & 1 & 12 & $0.70(0.09,5.43)$ & $0.91(0.11,7.35)$ & & \\
\hline Def 16 & 0 & 3 & & & & \\
\hline Def17 & 3 & 32 & $0.79(0.24,2.59)$ & $1.13(0.34,3.80)$ & & \\
\hline \multirow[t]{3}{*}{ Def18 } & 5 & 37 & $1.15(0.45,2.93)$ & $1.71(0.66,4.46)$ & & \\
\hline & & & & & $T$ & $T$ \\
\hline & & & & & .2 & 5 \\
\hline
\end{tabular}

Figure 1 Crude and adjusted HR (aHR) estimates for Fournier's gangrene for initiation of sodium-glucose cotransporter-2 inhibitors (SGLT2i) compared with initiation of dipeptidyl peptidase-4 inhibitor (DPP4i) (A) and all other non-SGLT2i antihyperglycemic medications (B), 365-day washout period, as-treated analysis. Key to outcome definitions: Def1: Diagnosis list 1 (any setting)*; Def2: Diagnosis list 1 (inpatient only); Def3: Diagnosis list 2 (any setting)†; Def4: Diagnosis list 2 (inpatient only); Def5: Diagnosis list 3 (any setting)ł; Def6: Diagnosis list 3 (inpatient only); Def7: Diagnosis list 1 (inpatient only), plus either systemic antibiotics, debridement, or surgery within 7 days; Def8: Diagnosis list 1 (inpatient only), plus systemic antibiotics and debridement and surgery within 7 days; Def9: Diagnosis list 2 (inpatient only), plus either systemic antibiotics, debridement, or surgery within 7 days; Def10: Diagnosis list 2 (inpatient only), plus systemic antibiotics and debridement and surgery within 7 days; Def11: Diagnosis list 3 (inpatient only), plus either systemic antibiotics, debridement, or surgery within 7 days; Def12: Diagnosis list 3 (inpatient only), plus systemic antibiotics and debridement and surgery within 7 days; Def13: Diagnosis list 1 (inpatient only), plus systemic antibiotics and either debridement or surgery within 7 days; Def14: Diagnosis list 2 (inpatient only), plus systemic antibiotics and either debridement or surgery within 7 days; Def15: Diagnosis list 3 (inpatient only), plus systemic antibiotics and either debridement or surgery within 7 days; Def16: Diagnosis list 1 (inpatient only), plus either debridement or surgery within 7 days; Def17: Diagnosis list 2 (inpatient only), plus either debridement or surgery within 7 days; Def18: Diagnosis list 3 (inpatient only), plus either debridement or surgery within 7 days. *Diagnosis list 1: 608.83, N49.3; †Diagnosis list 2: 608.83, N49.3, 785.4, 616.3, 616.4, N75.1, N76.4, 196; łDiagnosis list 3: 608.83, 608.4, N49.3, N50.1, 785.4, 616.3, 616.4, N75.1, N76.4, 196. Outcome definitions 8, 10, and 12 yielded 0 observed outcomes and are not shown in Figure.

\section{DISCUSSION}

Using a state-of-the-art ACNU study design for real-world evidence, we observed an increased risk of FG for SGLT2i initiators compared with initiators of a broad assortment of non-SGLT2i antihyperglycemic medications. However, estimates were imprecise due to the low number of observed events. These estimates appear to corroborate evidence behind the FDA's initial warning, but also demonstrate the importance of considering the most appropriate active comparator when conducting comparative safety studies.
When compared with initiators of DPP4i, the most commonly prescribed, branded second-line antihyperglycemic medication class, ${ }^{29}$ SGLT2i initiation did not appear to be associated with an increased risk of FG. The DPP4i comparator reflects the clinical decision between prescribing two similar branded second-line antihyperglycemic medications, whereas the non-SGLT2i comparator group contains a more heterogeneous set of medications including first-line metformin and third-line insulin as well as generic medications. Our results in the 
DPP4i comparison, which we believe to be the most relevant active-comparator class of glucose-lowering drugs to SGLT2i, are also corroborated by a recent meta-analysis of randomized trials ${ }^{30}$ as well as a recent commentary ${ }^{12}$ both of which addressed the topic of FG risk and concluded no increased risk for SGLT2i users.

Our results also highlight the important role of outcome classification and confounding control decisions in the setting of very rare outcomes. Although our primary outcome definition aimed to maximize specificity, the precision of resulting estimates was low due to only six observed events from 2013 to 2017 in the DPP4i comparison. This likely contributed to differences between crude and adjusted HR estimates; this difference decreased for higher-sensitivity outcome definitions. Furthermore, the ICD-9 diagnosis code 608.83 ('vascular disorders, including Fournier's disease') is non-specific to FG and can include hematoma of the scrotum, testicles, and seminal vesicle. However, this is the best available ICD-9 code and maps directly to the ICD-10 diagnosis for FG, $\mathrm{N} 49.3$, and has been used in previous studies of FG. ${ }^{245}$ Finally, the observational design of this study precluded our ability to control for unmeasured confounding, although the ACNU study design helps to minimize the potential for unmeasured confounding by implicitly controlling for confounding by indication. ${ }^{13}$

This study fills an important evidence gap regarding FG risk with SGLT2i use conducted using population-level data. The cohort study design allows for measurement of incidence, which was lacking in prior FAERS analyses. The two-prescription exposure definition increases confidence that patients are actually taking study drugs compared with cohorts requiring just a single prescription. Finally, the range of outcome definitions yielded consistent conclusions.

\section{CONCLUSIONS}

No evidence of increased risk of FG associated with SGLT2i was observed in the most relevant comparison with the commonly used, branded second-line antihyperglycemic agent, DPP4i. However, uncertainty remains based on potentially higher risk in the broader comparison with all non-SGLT2i antihyperglycemic medications and the low absolute incidence of FG.

Contributors JYY designed the study, performed the analyses, interpreted the results and drafted the manuscript. TW contributed to study design decisions, interpreted the results and edited the manuscript. VP performed data extraction and provided data analysis support and verification. JBB provided clinical oversight and expertise, contributed to study design decisions, interpreted the results and edited the manuscript. TS provided methods oversight and expertise, contributed to study design decisions, interpreted the results and edited the manuscript. JYY served as the guarantor.

Funding This research was supported, in part, by grants from the National Institutes of Health (R01 AG056479, T32 DK007634, UL1TR002489) and from the University of North Carolina Royster Society of Fellows. The database infrastructure used for this project was funded by the Department of Epidemiology, UNC Gillings School of Global Public Health; the Cecil G Sheps Center for Health Services Research, UNC; the CER Strategic Initiative of UNC's Clinical Translational Science Award (UL1TR002489); and the UNC School of Medicine.
Competing interests VP: receives salary support from the National Institute on Aging (R01 AG056479), National Institutes of Health (NIH) (R01 HL118255), and the National Center for Advancing Translational Sciences (NCATS, UL1TR002489), NIH. JBB: outside of the submitted work, JBB reports contracted consulting fees and associated travel support paid to his employer from Adocia, AstraZeneca, Dance Biopharm, Dexcom, Eli Lilly, Fractyl, Gl Dynamics, Intarcia Therapeutics, Lexicon, MannKind, Metavention, NovaTarg, Novo Nordisk, Orexigen, PhaseBio, Sanofi, Senseonics, vTv Therapeutics, and Zafgen; grants and related travel support from AstraZeneca, Eli Lilly, Intarcia Therapeutics, Johnson \& Johnson, Lexicon, Medtronic, Novo Nordisk, Sanofi, Theracos and vTv Therapeutics; personal fees from Cirius Therapeutics, CSL Behring; stock or stock options from Mellitus Health, PhaseBio, Stability Health, and Pendulum Health; grants from the US National Institutes of Health (UL1TR002489, U01DK098246, UC4DK108612, U54DK118612), PCORI and American Diabetes Association. TS: receives investigator-initiated research funding and support as principal investigator (R01 AG056479) from the National Institute on Aging (NIA), and as coinvestigator (R01 CA174453, R01 HL118255, R21-HD080214), National Institutes of Health (NIH). He also receives salary support as Director of Comparative Effectiveness Research (CER), NC TraCS Institute, UNC Clinical and Translational Science Award (UL1TR002489), the Center for Pharmacoepidemiology (current members: GlaxoSmithKline, UCB BioSciences, Merck, Takeda), from pharmaceutical companies (Amgen, AstraZeneca, Novo Nordisk), and from a generous contribution from Dr Nancy A Dreyer to the Department of Epidemiology, University of North Carolina at Chapel Hill. TS does not accept personal compensation of any kind from any pharmaceutical company. He owns stock in Novartis, Roche, BASF, AstraZeneca, and Novo Nordisk.

\section{Patient consent for publication Not required.}

Ethics approval The study was determined to be exempt from full Institutional Review Board review by the University of North Carolina at Chapel Hill.

Provenance and peer review Not commissioned; externally peer reviewed.

Data availability statement Data may be obtained from a third party and are not publicly available. All data relevant to the study are included in the article. The attached study uses administrative claims data from the MarketScan Commercial Claims and Encounters (CCAE) database, to which UNC-Chapel Hill holds a data use agreement. Under this data use agreement, aggregate-level summaries and results are allowed to be published and shared with external parties, but not the raw de-identified patient-level claims data.

Open access This is an open access article distributed in accordance with the Creative Commons Attribution Non Commercial (CC BY-NC 4.0) license, which permits others to distribute, remix, adapt, build upon this work non-commercially, and license their derivative works on different terms, provided the original work is properly cited, appropriate credit is given, any changes made indicated, and the use is non-commercial. See: http://creativecommons.org/licenses/by-nc/4.0/.

\section{ORCID iDs}

Jeff Yufeng Yang http://orcid.org/0000-0002-0817-983X

Tiansheng Wang http://orcid.org/0000-0002-0980-8896

John B Buse http://orcid.org/0000-0002-9723-3876

Til Stürmer http://orcid.org/0000-0002-9204-7177

\section{REFERENCES}

1 FDA. Fda warns about rare occurrences of a serious infection of the genital area with SGLT2 inhibitors for diabetes, 2018. Available: https://www.fda.gov/Drugs/DrugSafety/ucm617360.htm

2 Sorensen MD, Krieger JN. Fournier's gangrene: epidemiology and outcomes in the general US population. Urol Int 2016;97:249-59.

3 Kranz J, Schlager D, Anheuser P, et al. Desperate need for better management of Fournier's Gangrene. Cent Eur J Urol 2018;71:360-5

4 Sorensen MD, Krieger JN, Rivara FP, et al. Fournier's gangrene: management and mortality predictors in a population based study. $J$ Urol 2009;182:2742-7.

5 Sorensen MD, Krieger JN, Rivara FP, et al. Fournier's gangrene: population based epidemiology and outcomes. Journal of Urology 2009;181:2120-6.

6 Singh A, Ahmed K, Aydin A, et al. Fournier's gangrene. A clinical review. Arch Ital Urol Androl 2016;88:157-64.

7 Lee BY. How one class of diabetes medications may lead to flesheating genital infections, 2018. Available: https://www.forbes.com/ sites/brucelee/2018/09/01/how-one-class-of-diabetes-medicationsmay-lead-to-flesh-eating-genital-infections/\#75c509976193

8 Chi WC, Lim-Tio S. Fournier's syndrome: a life threatening complication of SGLT2 inhibition in poorly controlled diabetes 
mellitus (Poster \#265). Available: http://ads-adea-2016.m.asnevents. com.au/schedule/session/9239/abstract/36604

9 Kumar S, Costello AJ, Colman PG. Fournier's gangrene in a man on empagliflozin for treatment of Type 2 diabetes. Diabet. Med. 2017;34:1646-8.

10 Onder CE, Gursoy K, Kuskonmaz SM, et al. Fournier's gangrene in a patient on dapagliflozin treatment for type 2 diabetes. $J$ Diabetes 2019;11:348-50.

11 Bersoff-Matcha SJ, Chamberlain C, Cao C, et al. Fournier gangrene associated with sodium-glucose cotransporter-2 inhibitors: a review of spontaneous postmarketing cases. Ann Intern Med 2019;170:764-9.

12 Bloomgarden Z, Einhorn D, Grunberger G, Handelsman Y, et al. Fournier's gangrene and sodium-glucose cotransporter 2 inhibitors: is there a causal association? J Diabetes 2019;11:340-1.

13 Lund JL, Richardson DB, Stürmer T. The active comparator, new user study design in pharmacoepidemiology: historical foundations and contemporary application. Curr Epidemiol Rep 2015;2:221-8.

14 Mosley JF, Smith L, Everton E, et al. Sodium-Glucose linked transporter 2 (SGLT2) inhibitors in the management of type-2 diabetes: a drug class overview. Pharm Ther 2015;40:451-62.

15 Agarwal S, Pitcavage JM, Sud K, et al. Burden of readmissions among patients with critical limb ischemia. J Am Coll Cardiol 2017;69:1897-908.

16 Brennan MB, Allen GO, Ferguson PD, et al. The association between geographic density of infectious disease physicians and limb preservation in patients with diabetic foot ulcers. Open Forum Infect Dis 2017;4:1-8.

17 Turley RS, Mi X, Qualls LG, et al. The Effect of Clinical Care Location on Clinical Outcomes After Peripheral Vascular Intervention in Medicare Beneficiaries. JACC: Cardiovascular Interventions 2017;10:1161-71.

18 Eggers PW, Gohdes D, Pugh J. Nontraumatic lower extremity amputations in the Medicare end-stage renal disease population. Kidney Int 1999;56:1524-33.
19 Goldberg JB, Goodney PP, Cronenwett JL, et al. The effect of risk and race on lower extremity amputations among Medicare diabetic patients. J Vasc Surg 2012;56:1663-8.

20 Martinson M, Martinson N. A comparative analysis of skin substitutes used in the management of diabetic foot ulcers. $J$ Wound Care 2016;25:S8-17.

21 McEwen LN, Ylitalo KR, Munson M, et al. Foot complications and mortality: results from translating research into action for diabetes (triad). J Am Podiatr Med Assoc 2016;106:7-14.

22 Medhekar AN, Mix DS, Aquina CT, et al. Outcomes for critical limb ischemia are driven by lower extremity revascularization volume, not distance to hospital. J Vasc Surg 2017;66:476-87.

23 Newhall K, Spangler E, Dzebisashvili N, et al. Amputation rates for patients with diabetes and peripheral arterial disease: the effects of race and region. Ann Vasc Surg 2016;30:292-8.

24 Newhall K, Stone D, Svoboda R, et al. Possible consequences of regionally based bundled payments for diabetic amputations for safety net hospitals in Texas. J Vasc Surg 2016;64:1756-62.

25 Sargen MR, Hoffstad O, Margolis DJ. Geographic variation in Medicare spending and mortality for diabetic patients with foot ulcers and amputations. J Diabetes Complications 2013;27:128-33.

26 CMS. 2018 ICD-10 CM and GEMs. Available: https://www.cms. gov/Medicare/Coding/ICD10/2018-ICD-10-CM-and-GEMs.htm [Accessed 5 May 2019].

27 Austin PC. Some methods of propensity-score matching had superior performance to others: results of an empirical investigation and Monte Carlo simulations. Biom J 2009;51:171-84.

28 Sato T, Matsuyama Y. Marginal structural models as a tool for standardization. Epidemiology 2003;14:680-6.

29 Montvida O, Shaw J, Atherton JJ, et al. Long-term trends in antidiabetes drug usage in the U.S.: real-world evidence in patients newly diagnosed with type 2 diabetes. Diabetes Care 2018;41:69-78.

30 Silverii GA, Dicembrini I, Monami M, et al. Fournier's gangrene and sodium-glucose co-transporter-2 inhibitors: a meta-analysis of randomized controlled trials. Diabetes Obes Metab 2019. doi:10.1111/dom.13900. [Epub ahead of print: 21 Oct 2019]. 